Background Five hundred and nine patients, receiving moderate to high doses of oral corticosteroids (equivalent to $7.5 \mathrm{mg}$ prednisone daily or greater), were enrolled in two studies to examine the effect of risedronate.

Objectives We report here on the results of the group of postmenopausal women in these studies $(n=255)$.

Methods Patients in these studies were randomised to receive placebo, or risedronate (RIS) $2.5 \mathrm{mg}$, or RIS $5 \mathrm{mg}$ for 1 year. Patients were supplemented with daily elemental calcium (500$1000 \mathrm{mg}$ ) and the majority also received supplemental vitamin D (400 IU). Spinal BMD was measured every 3 months; spinal radiographs were taken at baseline and 1 year.

Results In this group of postmenopausal women, RIS $5 \mathrm{mg}$ significantly increased spinal BMD by $2.6 \%$ vs placebo ( $\mathrm{p}$ less than 0.01 ). Nine out of 56 placebo patients had a new vertebral fracture compared with 3 out of 62 in the RIS $5 \mathrm{mg}$ treated group. This vertebral fracture risk reduction was $73 \%(\mathrm{p}=0.050)$. Effects of $2.5 \mathrm{mg}$ were consistent with these results.

Conclusion In summary, this study demonstrates that risedronate is effective in maintaining or increasing bone mass and reducing the risk of new vertebral fractures in postmenopausal women on corticosteroid therapy. These findings are consistent with risedronate effects observed in large, 3 year studies in women with confirmed postmenopausal osteoporosis.

\section{SAT0171 EVALUTION OF PULMONARY FUNCTION IN PATIENTS WITH OSTEOPOROSIS}

J Natour, IJ Lombardi, LM Oliveira, AF Mayer, JR Jardim. Departamento de Medicina, Universidade Federal de São Paulo, São Paulo, Brasil

10.1136/annrheumdis-2001.658

Background By and large vertebral osteoporotic fractures result, in increased kyphosis angle. This alteration could led to disturbance in the pulmonary function. Spinal deformity is associated with alterations in pulmonary function.

Objectives Our objective was to evaluate the pulmonary function in osteoporotic patients.

Methods Fifteen women with osteoporosis and vertebral fracture (G1), 20 women with osteoporosis without vertebral fracture (G2) and 20 mormal women (G3) were selected. All performed spirometry in the Viatrace-130 SL spirometer.

Results Our results showed that women with osteoporosis and vertebral fracture had increased kyphosis angle (median $=60$ degrees), decreased forced vital capacity (G1 vs. G2, p = 0.020; G1 vs. G3, p = 0.039) and forced wxpiratory volume in the first second (FEV1)(G1 vs. G2, p = 0.008; G1 vs. G3, p = 0.014). When compared with women with no vertebral fracture and no osteoporosis there wasn 't difference. There was a negative correlation between thoracic kyphosis and the predicted value of expiratory forced volume in one second $(p=0.003)$. Since 55 degrees of thoracic kyphosis the pulmonary function had showed compromised $(\mathrm{p}=0.021)$.

Conclusion We conclude that women with vertebral fracture have an increase of their kyphosis angle and a decrease of pulmonary function.
SAT0172 OSTEOPOROSIS PROPHYLAXIS IN PATIENTS RECEIVING CORTICOSTEROIDS

RJ Davies, T Walton, M Carulli, G Kingsley, V Hajela. Rheumatology Department, University Hospital Lewisham, London, UK

10.1136/annrheumdis-2001.659

Background Approximately $2 \%$ of all general medical outpatients are taking corticosteroids. ${ }^{1}$ The link between corticosteroid consumption and osteoporosis with subsequent fracture is strong $^{2}$ and national guidelines have been drawn up for the prevention of corticosteroid induced osteoporosis (CSIO). ${ }^{3}$

Objectives To determine by means of re-audit whether management of CSIO improved following two education sessions and the distribution of guidelines to all general medical physicians, after the initial audit in July 1997.

Methods The standard used was the National Osteoporosis Society (NOS) guidelines for the management of CSIO. The notes of all patients attending rheumatology, gastroenterology and respiratory clinics at University Hospital Lewisham during July 1997 for the initial audit and April and May 2000 for the re-audit were examined. Education and guidelines about CSIO were given to all relevant departments and a re-audit undertaken 2 years later.

Results HIGH RISK GROUPS; age $>65$ years and on $>7.5$ $\mathrm{mg} / \mathrm{d}$ prednisolone or $<65$ years and on $>15 \mathrm{mg} / \mathrm{d}$ prednisolone.

Initial audit 67\% (57/85) were in this group, 5.2\% had lifestyle advice, $15.7 \% \quad \mathrm{BMD}$ and $43.8 \%$ were prescribed prophylaxis.

Re-audit $46.2 \%(37 / 80)$ were in this group, $24.3 \%$ had lifestyle advice, $51.3 \%$ BMD and $67.6 \%$ were prescribed prophylaxis.

MEDIUM RISK GROUPS; age $<65$ years, prednisolone dose $>7.5 \mathrm{mg} / \mathrm{d}<15 \mathrm{mg} / \mathrm{d}$.

Initial audit 7\% (6/85) were in this group, $16.6 \%$ had lifestyle advice, $50 \%$ BMD and $66.6 \%$ were receiving prophylaxis.

Re-audit $32.5 \%(26 / 80)$ were in this group, $30.8 \%$ had lifestyle advice, 50\% BMD and $42.3 \%$ were receiving prophylaxis.

LOW RISK GROUPS; prednisolone $<7.5 \mathrm{mg} / \mathrm{d}$.

Initial audit $25.8 \%(22 / 85)$ were in this group, none had received lifestyle advice, $22.7 \% \mathrm{BMD}$ and $27.2 \%$ were receiving prophylaxis.

Re-audit 21.2\% (17/80) were in this group, none received lifestyle advice, $23.5 \% \mathrm{BMD}$ and $52.9 \%$ were receiving prophylaxis.

Conclusion Education and distribution of NOS guidelines lead to an increase in the number of high risk patients receiving appropriate treatment.

The percentage of patients receiving prophylaxis in July 1997 was $41.1 \%$ and $56.2 \%$ in the re-audit.

Clarity is needed in the management of low risk groups.

Patients receiving lifestyle advice remains unacceptably low.

There is a reluctance to prescribed calcium and vitamin D to medium risk patients and those awaiting DEXA scan results.

\section{REFERENCES}

1 Bell R, Carr A, Thompson PW. Managing corticosteroid induced osteoporosis. J $R$ Coll Phys. 1997;31:158-61

2 American College of Rheumatology Task Force on Osteoporosis Guidelines. Recommendations for the prevention and treatment of glucocorticoid induced osteoporosis. Arthritis Rheumatol. 1996;39:1791-801

3 Eastell $R$, et al. A UK Consensus Group on management of glucocorticoid induced osteoporosis: an update. J Intern Med. 1998;244:271-92 